\title{
ON THE EXTREMAL SOLUTIONS OF SEMILINEAR ELLIPTIC PROBLEMS
}

\author{
LAMIA BEN CHAABANE
}

Received 1 March 2004

We investigate here the properties of extremal solutions for semilinear elliptic equation $-\Delta u=\lambda f(u)$ posed on a bounded smooth domain of $\mathbb{R}^{n}$ with Dirichlet boundary condition and with $f$ exploding at a finite positive value $a$.

\section{Introduction}

We consider the following semilinear elliptic problem:

$\left(P_{\lambda}\right)$

$$
\begin{gathered}
-\Delta u=\lambda f(u) \quad \text { in } \Omega, \\
u>0 \quad \text { in } \Omega, \\
u=0 \quad \text { on } \partial \Omega,
\end{gathered}
$$

where $\lambda>0, \Omega \subset \mathbb{R}^{n}$ is a bounded smooth domain and $f$ satisfies the following condition:

(H) $f$ is a $C^{2}$ positive nondecreasing convex function on $[0, \infty)$ such that

$$
\lim _{t \rightarrow+\infty} \frac{f(t)}{t}=+\infty
$$

It is well known that under this condition $(H)$, there exists a critical positive value $\lambda^{*} \in$ $(0, \infty)$ for the parameter $\lambda$ such that the following holds.

$\left(C_{1}\right)$ For any $\lambda \in\left(0, \lambda^{*}\right)$, there exists a positive, minimal, classical solution $u_{\lambda} \in C^{2}(\bar{\Omega})$. The function $u_{\lambda}$ is minimal in the following sense: for every solution $u$ of $\left(P_{\lambda}\right)$, we have $u_{\lambda} \leq u$ on $\Omega$. In addition, the function $\lambda \mapsto u_{\lambda}$ is increasing and $\lambda_{1}\left(-\Delta-\lambda f^{\prime}\left(u_{\lambda}\right)\right)>0$, for example, for any $\varphi \in H_{0}^{1}(\Omega) \backslash\{0\}$,

$$
\lambda \int_{\Omega} f^{\prime}\left(u_{\lambda}\right) \varphi^{2} d x<\int_{\Omega}|\nabla \varphi|^{2} d x .
$$

$\left(C_{2}\right)$ For any $\lambda>\lambda^{*}$, there exists no classical solution for $\left(P_{\lambda}\right)$. 
2 On the extremal solutions of semilinear elliptic problems

When $\lambda$ tends to $\lambda^{*}$,

$$
u^{*}=\lim _{\lambda \rightarrow \lambda^{*}} u_{\lambda}
$$

always exists by the monotonicity of $u_{\lambda}$. In [3], Brezis et al. have introduced a notion of weak solution as follows: we say $u$ is a weak solution for $\left(P_{\lambda}\right)$ if $u \in L^{1}(\Omega), u \geq 0$, $f(u) \delta \in L^{1}(\Omega)$ with $\delta(x)=\operatorname{dist}(x, \partial \Omega)$, and

$$
\int_{\Omega} u(-\Delta \xi) d x=\lambda \int_{\Omega} f(u) \xi d x
$$

for all $\xi \in C^{2}(\bar{\Omega}),\left.\xi\right|_{\partial \Omega}=0$. They then proved the following.

$\left(C_{3}\right) u^{*}$ is always a weak solution of the problem $\left(P_{\lambda^{*}}\right)$, and for $\lambda>\lambda^{*}$ no solution exists even in the weak sense.

Later, Martel proved in [6] that $u^{*}$ is the unique weak solution of $\left(P_{\lambda^{*}}\right)$, the so called extremal solution.

The typical examples are when the nonlinearity of $f$ is either exponential $f(u)=e^{u}$ or power-like $f(u)=(1+u)^{p}, p>1$ (see $\left.[4,5,7]\right)$. For $f(u)=e^{u}, u^{*}$ is smooth when $n \leq 9$, if $n \geq 10, u^{*}=-2 \ln |x|$ is the extremal solution on $B_{1}(0)$. When $f(u)=(1+u)^{p}$, if $n<n_{p}=6+4(1+\sqrt{p(p-1)}) /(p-1), u^{*}$ is regular, and for $n \geq n_{p}, u^{*}=|x|^{-2 /(p-1)}-1$ is the extremal solution on $B_{1}(0)$. An immediate consequence is that with any $p>1$ and $n \leq 10, u^{*}$ is a smooth solution. It is natural to ask the following question: for small dimension $n$, is $u^{*}$ always a classical solution for any function $f$ satisfying $(H)$ and any domain $\Omega \subset \mathbb{R}^{n}$ ? Nedev in [9] and Ye and Zhou in [10] had given some partial answers to this question.

THeORem 1.1 [9]. Suppose that $f$ satisfies $(H)$, then for $n=2$ or $3, u^{*}$ is always a classical solution. Moreover, when $n \geq 4, u^{*} \in L^{q}(\Omega)$, for any $q<n /(n-4)$ and $f\left(u^{*}\right) \in L^{q}(\Omega)$, for any $q<n /(n-2)$.

Theorem $1.2[10]$. Let $f$ verify $(H)$, rewrite $f(t)=f(0)+t e^{g(t)}$. Assume that there exists $t_{0}$ positive such that $t^{2} g^{\prime}(t)$ is nondecreasing in $\left[t_{0}, \infty\right)$, then for any $\Omega \subset \mathbb{R}^{n}$ with $n \leq 9, u^{*}$ is a classical solution.

On the other hand, Brezis and Vazquez have given a characterization of unbounded extremal solutions in $H_{0}^{1}(\Omega)$ as follows: if $v \in H_{0}^{1}(\Omega)$ is an unbounded weak solution of $\left(P_{\lambda}\right)$ with $\lambda>0$ and satisfying the stability condition

$$
\lambda \int_{\Omega} f^{\prime}(v) \varphi^{2} d x \leq \int_{\Omega}|\nabla \varphi|^{2} d x, \quad \forall \varphi \in C_{1}(\bar{\Omega}),\left.\varphi\right|_{\partial \Omega}=0
$$

then $\lambda=\lambda^{*}$ and $v=u^{*}$. They remarked also that there exist unbounded weak solutions which satisfy (1.6), but do not belong to $H_{0}^{1}(\Omega)$, and which are not extremal solutions.

In this paper, we investigate some similar problems with $f$ exploding at a finite positive value $a$. More precisely, let $f$ satisfy the following condition:

$\left(H^{\prime}\right) f$ is a $C^{1}$ positive, nondecreasing, convex function on $[0, a)$ with $a \in(0, \infty)$ and

$$
\lim _{t \rightarrow a^{-}} f(t)=+\infty
$$


We consider the following problem:

$\left(E_{\lambda}\right)$

$$
\begin{gathered}
-\Delta u=\lambda f(u) \quad \text { in } \Omega, \\
u \in(0, a] \quad \text { in } \Omega, \\
u=0 \quad \text { on } \partial \Omega .
\end{gathered}
$$

By the work of Mignot and Puel (see [7]), we have always a critical value $\lambda^{*} \in(0, \infty)$ such that for any $\lambda \in\left(0, \lambda^{*}\right)$, there exists a positive, minimal, classical solution $u_{\lambda} \in C^{2}(\bar{\Omega})$, that is, $u_{\lambda}<a$ in $(\bar{\Omega})$ and for $\lambda>\lambda^{*}$, no classical solution exists. The aim of this work is to study the propriety of the solution of $\left(E_{\lambda}\right)$ at the extremal value $\lambda=\lambda^{*}$ and to prove the nonexistence of weak solution when $\lambda>\lambda^{*}$. We define that $\omega$ is a weak solution of $\left(E_{\lambda}\right)$, if $\omega \in L^{1}(\Omega,[0, a])$ such that $f(\omega) \delta \in L^{1}(\Omega)$, and for all $\zeta \in C^{2}(\bar{\Omega})$, with $\zeta=0$ on $\partial \Omega$,

$$
-\int_{\Omega} \omega \Delta \zeta=\lambda \int_{\Omega} f(\omega) \zeta
$$

Similarly, we say that $\omega$ is a weak supersolution of $\left(E_{\lambda}\right)$, if $\omega \in L^{1}(\Omega,[0, a])$, such that $(\Delta \omega) \delta \in L^{1}(\Omega)$, and for all $\zeta \in C^{2}(\bar{\Omega}), \zeta \geq 0$ with $\zeta=0$ on $\partial \Omega$,

$$
-\int_{\Omega} \omega \Delta \zeta \geq \lambda \int_{\Omega} f(\omega) \zeta
$$

Our main results are the following.

Theorem 1.3. Given $f$ satisfying $\left(H^{\prime}\right)$, if $\lambda>\lambda^{*}$, then there is no weak solution of $\left(E_{\lambda}\right)$.

Theorem 1.4. The function $u^{*}=\lim _{\lambda \rightarrow \lambda^{*}} u_{\lambda}$ is the unique weak solution of $\left(E_{\lambda^{*}}\right)$. Moreover, for any $\varphi \in C^{1}(\bar{\Omega})$ with $\varphi=0$ on $\partial \Omega$,

$$
\lambda^{*} \int_{\Omega} f^{\prime}\left(u^{*}\right) \varphi^{2} d x \leq \int_{\Omega}|\nabla \varphi|^{2} d x
$$

Theorem 1.5. Assume that $v \in H_{0}^{1}(\Omega)$ is a weak solution of $\left(E_{\lambda}\right)$ for some $\lambda>0$, assume also that $\sup _{\Omega}(v)=a$ and

$$
\lambda \int_{\Omega} f^{\prime}(v) \varphi^{2} d x \leq \int_{\Omega}|\nabla \varphi|^{2} d x
$$

for all $\varphi \in C^{1}(\bar{\Omega}), \varphi=0$ on $\partial \Omega$, then $\lambda=\lambda^{*}$ and $v=u^{*}$.

\section{Proof of Theorem 1.3}

In fact, Theorem 1.3 is deduced from a general result, which is the following proposition.

Proposition 2.1. Given $g$ satisfying $\left(H^{\prime}\right)$, if there exists a weak solution $\omega$ of

$$
\begin{gathered}
-\Delta \omega=g(\omega) \quad \text { in } \Omega, \\
\omega=0 \quad \text { on } \partial \Omega,
\end{gathered}
$$


4 On the extremal solutions of semilinear elliptic problems

then, for any $\varepsilon \in(0,1)$, there exists a classical solution $\omega_{\varepsilon}$ of

$$
\begin{gathered}
-\Delta \omega_{\varepsilon}=(1-\varepsilon) g\left(\omega_{\varepsilon}\right) \quad \text { in } \Omega, \\
\omega_{\varepsilon}=0 \quad \text { on } \partial \Omega .
\end{gathered}
$$

For the proof of this result, we need the following lemmas which are proved in [3].

Lemma 2.2. Given $g \in L^{1}(\Omega, \delta(x) d x)$, there exists a unique $v \in L^{1}(\Omega)$ which is a weak solution of

$$
\begin{gathered}
-\Delta v=g \quad \text { in } \Omega, \\
v=0 \quad \text { on } \partial \Omega,
\end{gathered}
$$

where $\|v\|_{L^{1}} \leq C\|g\|_{L^{1}(\Omega, \delta(x) d x)}$, for some $C$ constant independent of $g$. In addition, if $g \geq 0$ a.e. in $\Omega$, then $v \geq 0$ a.e. in $\Omega$.

Lemma 2.3. Assume $g(0)>0$ and set

$$
h(u)=\int_{0}^{u} \frac{d s}{g(s)}
$$

for all $0 \leq u \leq a$. Let $\tilde{g}$ be a $C^{1}$ positive function on $[0, a)$ such that $\tilde{g} \leq g$ and $\tilde{g}^{\prime} \leq g^{\prime}$. Set

$$
\tilde{h}(u)=\int_{0}^{u} \frac{d s}{\tilde{g}(s)}, \quad \Phi(u)=\tilde{h}^{-1}(h(u)),
$$

for all $u \in[0, a]$. Then,

(i) $\Phi(0)=0$ and $0 \leq \Phi(u) \leq u$ for all $0 \leq u \leq a$,

(ii) $\Phi$ is increasing, concave, and $\Phi^{\prime}(u) \leq 1$ for all $0 \leq u \leq a$,

(iii) $h(a)<\infty$ and $\Phi(a)<a$, if $\tilde{g} \neq \equiv$ in $[0, a]$.

Proof. It is easy to see that (i) and (iii) hold. We prove (ii), in fact $\Phi^{\prime}(u)=\tilde{g}(\Phi(u)) / g(u)>$ 0 , and

$$
\Phi^{\prime \prime}(u)=\frac{g(u) \tilde{g}^{\prime}(\Phi(u)) \Phi^{\prime}(u)-\tilde{g}(\Phi(u)) g^{\prime}(u)}{g(u)^{2}}=\frac{\tilde{g}(\Phi(u))\left(\tilde{g}^{\prime}(\Phi(u))-g^{\prime}(u)\right)}{g(u)^{2}} .
$$

Since $\tilde{g}^{\prime}(\Phi(u)) \leq g^{\prime}(\Phi(u)) \leq g^{\prime}(u)$, it follows that $\Phi$ is concave, which completes the proof.

Proof of Proposition 2.1 and Theorem 1.3. Choosing $\tilde{g}=(1-\varepsilon) g$ in Lemma 2.3 and denote by $v=\Phi(\omega)$, where $\omega$ is the weak solution of (2.1) and using an approximating 
argument for $\omega$, we get

$$
\begin{aligned}
-\int_{\Omega} v \Delta \zeta & =-\int_{\Omega} \Phi(\omega) \Delta \zeta=-\int_{\Omega} \Delta \Phi(\omega) \zeta=-\int_{\Omega}\left[\Phi^{\prime}(\omega) \Delta \omega+\Phi^{\prime \prime}(\omega)|\nabla \omega|^{2}\right] \zeta \\
& \geq \int_{\Omega} \Phi^{\prime}(\omega) g(\omega) \zeta=\int_{\Omega} \tilde{g}(\Phi(\omega)) \zeta=\int_{\Omega}(1-\varepsilon) g(v) \zeta
\end{aligned}
$$

for any $\zeta \in C^{1}(\bar{\Omega}), \zeta \geq 0$ with $\zeta=0$ on $\partial \Omega$. Hence, $v$ is a weak supersolution of (2.2). The result of Proposition 2.1 follows by standard barrier method as follows. We define a sequence $\left(\omega_{k}\right)_{k \geq 0}$ by

$$
\begin{gathered}
-\Delta \omega_{k+1}=(1-\varepsilon) g\left(\omega_{k}\right) \quad \text { in } \Omega, \\
\omega_{k+1}=0 \quad \text { on } \partial \Omega,
\end{gathered}
$$

for $k \in \mathbb{N}$, with $\omega_{0}=v$. Using Lemma 2.2 , it is easy to check that $\omega_{k} \geq \omega_{k+1} \geq 0$, for all $k \in \mathbb{N}$, so the sequence $\omega_{k}$ is nonincreasing and converges in $L^{1}(\Omega)$ to a weak solution $u$ of (2.2). Since $\sup _{\Omega}(u) \leq \sup _{\Omega}(v)<a, u$ is a classical solution, Proposition 2.1 is proved. Theorem 1.3 is deduced by taking $g=\lambda f$ in Proposition 2.1. For any $\lambda>\lambda^{*}$, let $\varepsilon \in(0,1)$ such that $\lambda^{*}<(1-\varepsilon) \lambda<\lambda$, since there is no classical solution of

$$
\begin{gathered}
-\Delta \omega_{\varepsilon}=(1-\varepsilon) \lambda f\left(\omega_{\varepsilon}\right) \quad \text { in } \Omega, \\
\omega_{\varepsilon}=0 \quad \text { on } \partial \Omega,
\end{gathered}
$$

it follows by Proposition 1.3 that there is no weak solution of $\left(E_{\lambda}\right)$.

\section{Proof of Theorem 1.4}

We know that $u^{*}$ is the increasing limit of classical solution $u_{\lambda}$ with positive first eigenvalue, that is, for any $\varphi \in C^{1}(\bar{\Omega})$ with $\varphi=0$ on $\partial \Omega$,

$$
\lambda \int_{\Omega} f^{\prime}\left(u_{\lambda}\right) \varphi^{2} d x \leq \int_{\Omega}|\nabla \varphi|^{2} d x
$$

Passing to the limit, the inequality (1.11) holds. To prove the uniqueness, we will in fact also prove a slightly stronger result.

Proposition 3.1. Let $v \in L^{1}(\Omega,[0, a])$ be a weak supersolution of $\left(E_{\lambda^{*}}\right)$, then $v=u^{*}$.

Proof. We proceed in two steps. First, we show that $v$ is a weak solution of $\left(E_{\lambda^{*}}\right)$. Next, we prove that if $v \neq u^{*}$, then we obtain a contradiction.

Step 1. Suppose that $v$ is not a weak solution of $\left(E_{\lambda^{*}}\right)$, then we can assume that there exists $\beta>0$ and $\xi_{0} \in C^{2}(\bar{\Omega}), \xi_{0} \geq 0$, with $\left.\xi_{0}\right|_{\partial \Omega}=0$ such that

$$
-\int_{\Omega} v \Delta \xi_{0}=\lambda^{*} \int_{\Omega} f(v) \xi_{0}+\beta
$$

it follows that there exists a nonnegative measure $\mu \neq \equiv$, with $\mu \delta$ bounded on $\Omega$, such that

$$
-\int_{\Omega} v \Delta \xi=\int_{\Omega}\left(\lambda^{*} f(v)+\mu\right) \xi
$$


6 On the extremal solutions of semilinear elliptic problems

for all $\xi \in C^{2}(\bar{\Omega})$ with $\left.\xi\right|_{\partial \Omega}=0$. Consider $\varphi$ and $\chi$, the solutions of

$$
\begin{array}{llll}
-\Delta \varphi=\mu & \text { in } \Omega, & \varphi=0 & \text { on } \partial \Omega, \\
-\Delta \chi=1 & \text { in } \Omega, & \chi=0 & \text { on } \partial \Omega .
\end{array}
$$

By $\mu \neq \equiv$, it follows from the properties of the Laplacian that there exists $\varepsilon>0$ such that $\varepsilon \chi \leq \varphi$. Set $z=v+\varepsilon \chi-\varphi \leq v$. Then, since $f$ is nondecreasing,

$$
-\int_{\Omega} z \Delta \xi=\int_{\Omega}\left(\lambda^{*} f(v)+\varepsilon\right) \xi \geq \int_{\Omega}\left(\lambda^{*} f(z)+\varepsilon\right) \xi
$$

for all $\xi \in C^{2}(\bar{\Omega}), \xi \geq 0$, with $\left.\xi\right|_{\partial \Omega}=0$. This means that $z$ is a weak supersolution for $-\Delta \omega=g(\omega)$, where $g(v)=\lambda^{*} f(v)+\varepsilon$. Using the proof of Proposition 2.1 and Lemma 2.3 with $\tilde{g}(v)=\lambda^{*} f(v)+\varepsilon / 2$, we can get a classical solution $v_{1}$ of

$$
\begin{gathered}
-\Delta v_{1}=\lambda^{*} f\left(v_{1}\right)+\left(\frac{\varepsilon}{2}\right) \quad \text { in } \Omega, \\
v_{1}=0 \quad \text { on } \partial \Omega .
\end{gathered}
$$

Moreover, there exists $\alpha>0$, such that $2 \alpha v_{1} \leq \varepsilon \chi$. Set $z=v_{1}+\alpha v_{1}-(\varepsilon / 2) \chi$. It is clear that $0<z \leq v_{1}$ and $z$ satisfies $-\Delta z \geq(1+\alpha) \lambda^{*} f\left(v_{1}\right) \geq(1+\alpha) \lambda^{*} f(z)$ in $\Omega$. Thus, the classical barrier method gives a solution of $\left(E_{(1+\alpha) \lambda^{*}}\right)$, which contradicts then the definition of $\lambda^{*}$, so $v$ is a solution of $\left(E_{\lambda^{*}}\right)$.

Step 2. Clearly, $v \geq u_{\lambda}$ for any $\lambda<\lambda^{*}$, hence $v \geq u^{*}$. Suppose that $v \neq \equiv u^{*}$, take $\Psi=f(v)-$ $f\left(u^{*}\right) \geq 0$, it is clear that $\Psi \delta \in L^{1}(\Omega)$. We have then $\Psi \not \equiv 0$, because otherwise $f(v)=$ $f\left(u^{*}\right)$ a.e. on $\Omega$, and Lemma 2.2 will give $v=u^{*}$ a.e. on $\Omega$. Let $g$ be the weak solution of

$$
\begin{gathered}
-\Delta g=\Psi \quad \text { in } \Omega, \\
g=0 \quad \text { on } \partial \Omega .
\end{gathered}
$$

By the maximum principle, we have $g \geq c \delta$ on $\Omega$ for some $c>0$. Hence,

$$
-\int_{\Omega}\left(v-u^{*}-\lambda^{*} g\right) \Delta \xi=0
$$

for all $\xi \in C^{2}(\bar{\Omega})$, with $\left.\xi\right|_{\partial \Omega}=0$. We obtain by Lemma 2.2 that $v-u^{*}=\lambda^{*} g \geq \lambda^{*} c \delta$ a.e. on $\Omega$, set $Z=\left(v+u^{*}\right) / 2$, then

$$
-\int_{\Omega} Z \Delta \xi=\frac{\lambda^{*}}{2} \int_{\Omega}\left(f(v)+f\left(u^{*}\right)\right) \xi=\lambda^{*} \int_{\Omega}(f(Z)+h) \xi>\lambda^{*} \int_{\Omega} f(Z) \xi
$$

for all $\xi \in C^{2}(\bar{\Omega}), \xi \geq 0$, with $\left.\xi\right|_{\partial \Omega}=0$, where $h$ is given by

$$
h=\frac{1}{2}\left(f(v)+f\left(u^{*}\right)\right)-f\left(\frac{v+u^{*}}{2}\right)=\frac{1}{2} \int_{u^{*}}^{v} d s \int_{\left(s+u^{*}\right) / 2}^{s} f^{\prime \prime}(\sigma) d \sigma .
$$


Clearly, $h \delta \in L^{1}(\Omega)$. Suppose first that $h \equiv 0$, then $f^{\prime \prime}(\sigma)=0$ if $\sigma \in\left[u^{*}, v\right]$, hence $f(\sigma)=$ $f(0)+f^{\prime}(0) \sigma$ on $\cup_{x \in \Omega}\left[u^{*}(x), v(x)\right]=\left[0, \sup _{\Omega} v\right]$, since $v>u^{*}$ in $\Omega$. Then, if $\sup _{\Omega} v=a$, we obtain a contradiction by (1.7), and if $\sup _{\Omega} v<a$, both $u^{*}$ and $v$ are classical solutions of a linear problem with $f(t)=A+B t$ for which the uniqueness is known (see, for instance, [8]). If $h \neq \equiv 0$, it follows that $Z$ is a strict supersolution of $\left(E_{\lambda *}\right)$ and we obtain also a contradiction by Step 1.

\section{Proof of Theorem 1.5}

Suppose that $\lambda<\lambda^{*}$. We observe that by a density argument, the inequality (1.12) holds for every $\Phi \in H_{0}^{1}(\Omega)$. Taking $\Phi=v-u_{\lambda}$ in (1.12), we get

$$
\lambda \int_{\Omega} f^{\prime}(v)\left(v-u_{\lambda}\right)^{2} d x \leq \int_{\Omega}\left|\nabla\left(v-u_{\lambda}\right)\right|^{2} d x=\lambda \int_{\Omega}\left[f(v)-f\left(u_{\lambda}\right)\right]\left(v-u_{\lambda}\right) d x
$$

that is,

$$
\lambda \int_{\Omega}\left[f(v)-f\left(u_{\lambda}\right)-f^{\prime}(v)\left(v-u_{\lambda}\right)\right]\left(v-u_{\lambda}\right) d x \geq 0
$$

Since $f$ is convex and $v \geq u_{\lambda}$, we get $f(v)=f\left(u_{\lambda}\right)+f^{\prime}(v)\left(v-u_{\lambda}\right)$ a.e. on $\Omega$. Hence, $f$ must be linear in the interval $\left[u_{\lambda}(x), v(x)\right]$ for a.e. $x \in \Omega$. If $v>u_{\lambda}$, we get that $f$ is linear in $\cup_{x}[u(x), v(x)]=\left[0, \sup _{\Omega} v\right)=[0, a)$, which contradicts $(1.7)$. So, $v=u_{\lambda}$, as $v$ is not a classical solution, we get a contradiction, so $\lambda=\lambda^{*}$. The similar argument with (1.11) shows that $v=u^{*}$.

\section{Application}

Now, we consider a special case $f(u)=1 /(1-u)^{p}$ with $p>0$ and $\Omega=B_{1}(0)$, this problem was studied by Brauner and Nicolaenko in $[1,2]$. When $p=1$, this equation appears as a limit of some problem of disruption in biochemistry; it allows then to justify some phenomenon in kinetic enzymatic and the kinetic of reactors associated to some limit coat. For $n \geq 2$, we know an explicit weak solution

$$
U(x)=1-|x|^{2 /(p+1)},
$$

which is obviously in $H_{0}^{1}(\Omega)$, it corresponds to the parameter value

$$
\lambda^{\#}(n, p)=\frac{2}{p+1}\left(n-\frac{2 p}{p+1}\right)>0 .
$$

The linearized operator is

$$
L_{\sharp} \Phi=-\Delta \Phi-\frac{2 p}{p+1}\left(n-\frac{2 p}{p+1}\right) \frac{\Phi}{r^{2}},
$$


where $r=|x|$. By Theorem 1.5, $U$ is the extremal solution if and only if for any $\Phi \in$ $H_{0}^{1}(\Omega)$,

$$
\frac{2 p}{p+1}\left(n-\frac{2 p}{p+1}\right) \int_{B} \frac{\Phi^{2}}{r^{2}} \leq \int_{B}|\nabla \Phi|^{2} d x
$$

Thanks to Hardy's inequality, this holds if and only if (see [4])

$$
\frac{2 p}{p+1}\left(n-\frac{2 p}{p+1}\right) \leq H=\frac{(n-2)^{2}}{4} \text {. }
$$

Thus, we have the following proposition.

Proposition 5.1. For any $p>0$, let

$$
n_{0}(p)=\frac{2}{p+1}[(3 p+1)+2 \sqrt{p(p+1)}] .
$$

Then,

(i) if $n \geq n_{0}(p), u^{*}(x)=1-|x|^{2 /(p+1)}$, and $\lambda^{*}=\lambda^{\#}$;

(ii) if $n<n_{0}(p), \lambda^{*}>\lambda^{\#}$ and $u^{*}$ is smooth.

Proof. By an easy computation, we have that $n \geq n_{0}(p)$ is equivalent to (5.5), so (i) is proved by Theorem 1.5. The proof of (ii) is given in [7].

We remark that when $p$ tends to $0, n_{0}(p)$ tends to 2 . So, for any $n \geq 3$, we can meet some nonlinearities $f$ (by choosing appropriate $p$ ) such that the extremal solution is no longer classical, this fact is different from the situation for $a=\infty$, if we compare with the results in $[9,10]$. Thus, a natural question is raised, for $f$ satisfying $\left(H^{\prime}\right)$ and $\Omega$ bounded smooth domain in $\mathbb{R}^{2}$, do we have always that $u^{*}$ is a classical solution?

\section{Acknowledgment}

The author would like to thank D. Ye and S. Baraket for bringing his attention to this problem and for insightful comments and assistance through this work.

\section{References}

[1] C.-M. Brauner and B. Nicolaenko, Sur une classe de problèmes elliptiques non linéaires, C. R. Acad. Sci. Paris Sér. A-B 286 (1978), no. 21, A1007-A1010 (French).

[2] _ Sur des problèmes aux valeurs propres non linéaires qui se prolongent en problèmes à frontière libre, C. R. Acad. Sci. Paris Sér. A-B 288 (1979), no. 2, A125-A127 (French).

[3] H. Brezis, T. Cazenave, Y. Martel, and A. Ramiandrisoa, Blow up for $u_{t}-\Delta u=g(u)$ revisited, Adv. Differential Equations 1 (1996), no. 1, 73-90.

[4] H. Brezis and J. L. Vázquez, Blow-up solutions of some nonlinear elliptic problems, Rev. Mat. Univ. Complut. Madrid 10 (1997), no. 2, 443-469.

[5] M. G. Crandall and P. H. Rabinowitz, Some continuation and variational methods for positive solutions of nonlinear elliptic eigenvalue problems, Arch. Ration. Mech. Anal. 58 (1975), no. 3, 207-218.

[6] Y. Martel, Uniqueness of weak extremal solutions of nonlinear elliptic problems, Houston J. Math. 23 (1997), no. 1, 161-168. 
[7] F. Mignot and J.-P. Puel, Sur une classe de problèmes non linéaires avec non linéairité positive, croissante, convexe, Comm. Partial Differential Equations 5 (1980), no. 8, 791-836 (French).

[8] P. Mironescu and V. D. Rădulescu, The study of a bifurcation problem associated to an asymptotically linear function, Nonlinear Anal. 26 (1996), no. 4, 857-875.

[9] G. Nedev, Regularity of the extremal solution of semilinear elliptic equations, C. R. Acad. Sci. Paris Sér. I Math. 330 (2000), no. 11, 997-1002.

[10] D. Ye and F. Zhou, Boundedness of the extremal solution for semilinear elliptic problems, Commun. Contemp. Math. 4 (2002), no. 3, 547-558.

Lamia Ben Chaabane: Département de Mathématiques, Faculté des Sciences de Tunis, Campus Universitaire, 2092 Tunis, Tunisia

E-mail address: lamia.benchaabane@fst.rnu.tn 


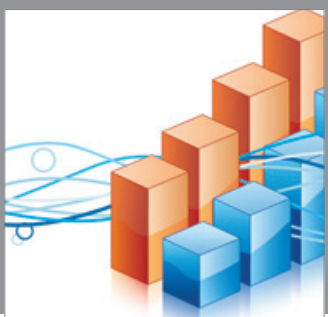

Advances in

Operations Research

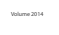

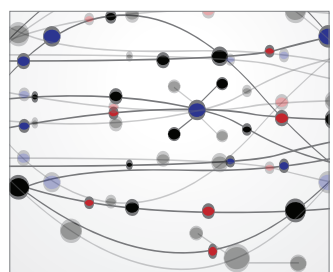

\section{The Scientific} World Journal
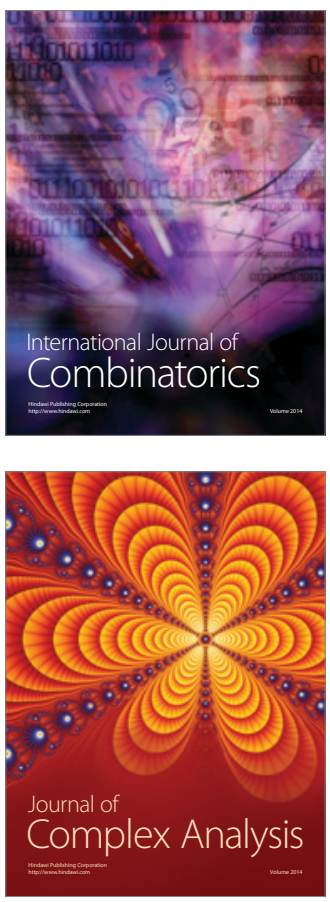

International Journal of

Mathematics and

Mathematical

Sciences
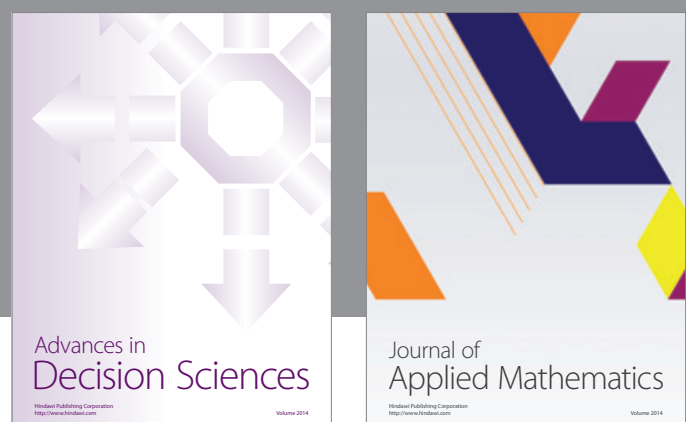

Journal of

Applied Mathematics
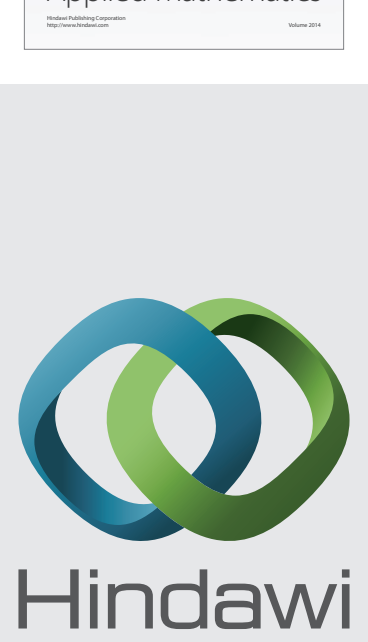

Submit your manuscripts at http://www.hindawi.com
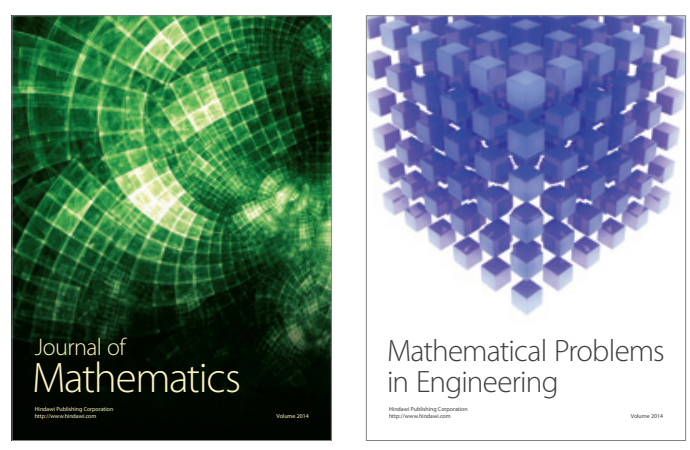

Mathematical Problems in Engineering
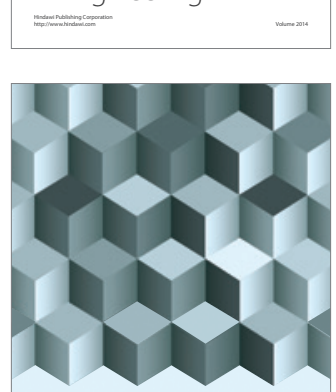

Journal of

Function Spaces
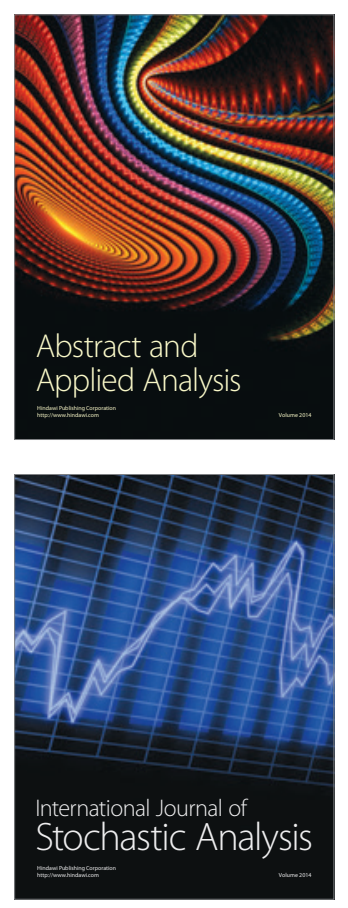

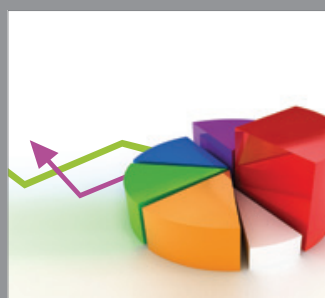

ournal of

Probability and Statistics

Promensencen
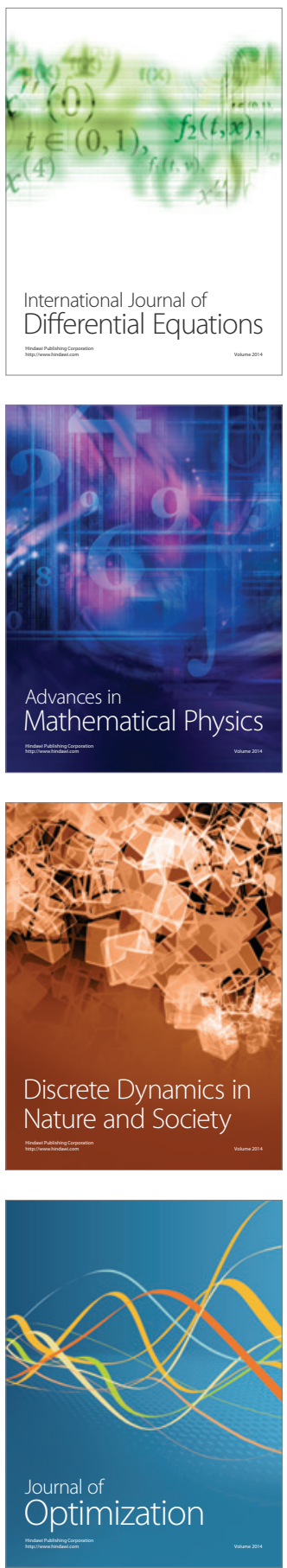